1 Occupancy-collection models: Towards bias-corrected modeling of species' distributions using unstructured occurrence data from museums and herbaria

3 Kelley D. Erickson*

4 Adam B. Smith

5 Center for Conservation and Sustainable Development, Missouri Botanical Garden, Saint Louis

6 MO 63110 USA

$7 \quad *$ Corresponding author: kerickson22@gmail.com

9 Abstract

10 The digitization of museum collections as well as an explosion in citizen science initiatives has

11 resulted in a wealth of data that can be useful for understanding the global distribution of

12 biodiversity, provided that the well-documented biases inherent in unstructured opportunistic

13 data are accounted for. While traditionally used to model imperfect detection using structured

14 data from systematic surveys of wildlife, occupancy-detection models provide a framework for

15 modelling the imperfect collection process that results in digital specimen data. In this study, we

16 explore methods for adapting occupancy-detection models for use with biased opportunistic

17 occurrence data from museum specimens and citizen science platforms using 7 species of

18 Anacardiaceae in Florida as a case study. We explored two methods of incorporating information

19 about collection effort to inform our uncertainty around species presence: (1) filtering the data to

20 exclude collectors unlikely to collect the focal species and (2) incorporating collection covariates

21 (collection type and history of previous detections) into a model of collection probability. We 
22 found that the best models incorporated both the background data filtration step as well as the

23 incorporation of collector covariates associated with the probability of collection. We found that

24 month, method of collection and whether a collector had previously collected the focal species

25 were important predictors of collection probability. Efforts to standardize meta-data associated

26 with data collection will improve efforts for modeling the spatial distribution of a variety of

27 species.

30 Understanding the extent of species ranges is a fundamental concern with many applications,

31 whether it is establishing areas of conservation concern (Brooks et al. 2004), identifying the

32 spread of invasive species (Václavík and Meentemeyer 2012, Veran et al. 2016, Briscoe et al.

33 2019), or predicting how species may respond to global change (Zurell et al. 2016, Scherrer et al.

34 2017, Graham et al. 2017, Requena-Mullor et al. 2019). Coupled with this growing urgency for

35 establishing species' ranges has been an explosion in the amount of occurrence data available

36 due to the rise in the digitization of museum specimens as well as the growth of citizen science

37 initiatives (Powney and Isaac 2015, Meineke et al. 2018). Originally collected for a wide variety

38 of intents and purposes, there are striking geographic and temporal biases in when and where

39 specimens are collected (Hijmans et al. 2000, Sólymos 2007, Meyer et al. 2016). Thus, much of

40 our understanding of the distribution of life on Earth comes from so-called "unstructured"

41 museum, herbarium, and citizen science data (in contrast to "structured" systematic sampling

42 such as survey or atlas data). 
43 To date, the most common method for utilizing unstructured data to recreate species'

44 distributions is to apply species distribution models (Peterson et al. 2011) (Figure 1a). Owing to

45 the numerous sources of biases and inaccuracies associated with occurrence data (Hijmans et al.

46 2000, Sólymos 2007, Meyer et al. 2016), there have been several approaches to correct for these

47 biases ranging from use of target-group background sites (occurrence records of other species

48 likely to have been collected along with the focal species as a proxy of effort, Ponder et al.

49 2001), systematic sub-sampling in geographic or environmental space, the use of bias files based

50 on proxies of search effort, and aggregating background points (Phillips et al. 2009, Boria et al.

51 2014, Fourcade et al. 2014, Varela et al. 2014, Vollering et al. 2019). In most of these

52 approaches, collection bias is assumed to be "canceled" out by contrasting biased occurrence

53 data with "filtered" background sites that are manipulated to have the same (presumed) bias as

54 the occurrences. However, the actual bias in unstructured data is rarely observed. As a result,

55 employing these methods is based on an assumption about the underlying bias.

Originally developed for systematic repeated-visit wildlife surveys, occupancy-detection models

57 (MacKenzie et al. 2002, 2003, 2018) explicitly account for the underlying processes which is an unknown latent state. When observers visit a site, the probability that they detect the focal species is a function of both the underlying reality of whether the site is occupied as well as the

61 probability of detecting the focal species if it is present. Detection can depend on a number of

62 covariates which can reflect, for example, time of year, observer experience, collection type

63 (e.g., museum specimen, photograph), and observer identity. Occupancy-detection models have

64 been increasingly used to analyze data from citizen-science projects, which often document 
attempt to report all species of a known taxon (e.g., Bled et al. 2013, Van Strien et al. 2013,

Berberich et al. 2016, Miller et al. 2016, Louvrier et al. 2018). These approaches assume that the data is structured in some way (i.e., involves defined surveying units with standardized instructions on how to collect and report data). Despite their utility for structured data, occupancy-detection models are challenging to apply to unstructured data because search effort

71 is rarely documented (although see Wolf et al. 2011). While the majority of digital occurrence data does not come from systematic sampling protocols, there is an associated wealth of often-overlooked data on how the specimen was collected on specimen labels that provides information about the collection process. Aside from simply identifying the species and where it was collected, specimen labels often also include the name of the collector and when it was collected. This type of information is commonly included in

77 specimen databases, but its potential for informing bias correction when modeling species Roberts et al. 2007) and thus collate unstructured data into a "structured" format for use in occupancy-detection modeling.

To use occupancy-detection models with unstructured occurrence data, careful decisions have to

83 be made about how to translate it into a format that is appropriate for the occupancy-detection

84 framework. One of the first considerations involves defining both the temporal and spatial scale of sampling. Typically, in studies involving structured survey data, whether they involve systematic wildlife surveys (e.g. MacKenzie et al. 2002) or atlas data (Bled et al. 2013, Altwegg

87 and Nichols 2019), the sampling unit is determined prior to the initiation of the study. When using digital occurrence records, the definition of surveys must be made retrospectively. While it 
would be possible to use coordinate-points associated with occurrences to define sites, the vast majority of specimens are only able to be geolocated to much larger geo-political units, such as counties or equivalent administrative units(c.f., Collins et al. 2017, Park and Davis 2017, Pender et al. 2019). Estimating the probability of detection requires that sites are visited more than once (MacKenzie et al. 2002), so the spatial and temporal scale of sampling should be carefully chosen to ensure that each sampling unit has multiple occurrence records within it. At the other extreme, if the temporal scale is too large, the assumption that sites are closed to changes in occupancy within the surveying period may be violated, in which case, either the estimated probability of occupancy should be thought of as a "usage probability" instead of true occupancy (MacKenzie et al. 2018), or a dynamic "multi-season" occupancy-detection model should be used (MacKenzie et al. 2003). Checklists can be assembled by collating the occurrence records made by a unique collector within the retrospectively-defined survey window of space and time, and then pre-existing methods of translating checklist data into detection histories can be used (Altwegg and Nichols 2019). When constructing checklists, duplicate specimens of the same species within the sampling unit by the same collector should be discarded. modeling species' distributions. As a case study illustrating our methods, we focus on seven species in the family Anacardiaceae (cashews and sumac) in the US state of Florida. For each

107 species, we collated metadata from occurrence records to reconstruct individual collectors' collection histories, the type of occurrence record being made (herbarium sheet or photograph), and their taxonomic focus, as well as information on the timing of collection within and across

110 years. Using this metadata, we were able to apply bias-correction frameworks from species 111 distribution modeling and occupancy-detection modeling (Fig. 1), alone and in combination. 
112 More generally, we demonstrate the potential for using oft-overlooked information in collection

113 records for accounting for bias in unstructured survey data. For simplicity, we focus here on the

114 single-season occupancy-detection model (sensu MacKenzie et al. 2002), although our approach

115 is amenable to any of the subsequent extensions of the basic occupancy-detection modeling

116 framework that have been developed since then, such as dynamic multi-season models

117 (MacKenzie et al. 2003), multi-species models (e.g. Van der Weyde et al. 2018), or models that

118 address the issue of misidentification (Miller et al. 2011).

119 MATERIAL AND METHODS

120 Overview

121 We compiled occurrence record data for species in the family Anacardiaceae and for all

122 Tracheophytes in the US state of Florida. For each species we constructed four models: (1) a full

123 model with unfiltered background (non-detection) data, (2) a full model with filtered data, (3) a

124 simple model with all data and (4) a simple model with filtered data. "Full" models incorporated 125 covariates related to the probability of detection, whereas "simple" models did not. "Unfiltered"

126 background data used data from all Tracheophytes as non-detections, whereas "filtered" data

127 represented a subset of these records selected such that the collectors were more likely to have

128 collected the focal species. We then evaluated the predictive accuracy of each model using cross-

129 validation. We use the term "botanical collector" to refer to a person or team of people who

130 collected a specimen in the field and deposited it into an herbarium, "citizen scientist" to a

131 person who contributed a record in a citizen scientist data portal (e.g., iNaturalist), and

132 "collector" to refer to either of these. 


\section{Data downloads and processing}

Occurrence records were converted to detection histories as follows. First, records with identical

We downloaded occurrence records for all Tracheophytes in Florida from the Global Biodiversity Information Facility (GBIF download doi: https://doi.org/10.15468/dl.zsgv2l and https://doi.org/10.15468/dl.tzxjrm) on 12 and 13 February 2019. Although traditionally a repository for only museum and herbarium records, in recent years GBIF has included "research grade" records from citizen science data portals, which in our case, included iNaturalist (www.inaturalist.org), naturegucker (naturgucker.de) and Questagame (https://collections.ala.org.au/public/show/dr1902). County names and collector names were cleaned and filtered using regular expressions in R and openRefine (Huynh and Mazzocchi 2019). As only $42 \%$ of our downloaded occurrence records had coordinates, occurrence records with coordinates were converted to county-level records by determining which county polygon each point fell within based on the Database of Global Administrative Areas, version 2.8 (GADM; www.gadm.org). Botanical collectors often create duplicate specimen sheets from the same individual which are sent to other institutions. To remove these duplicates from our analysis, we kept only the first occurrence of a species by a particular collector on a particular date. Finally, we removed records that were missing information on who collected the record, which species was recorded, and when the record was recorded, leaving us with 166,230 countylevel occurrence records of vascular plants in Florida. From these occurrence records, we selected all records for plants in the family Anacardiaceae, then of these retained only the seven species represented by $\geq 15$ non-duplicate occurrences (Table A1 in Appendix A) for our focal species. We then modeled the distributions of these seven species.

collectors, months, years and county of collection were grouped together and denoted 'collection 
157 event' $t$ (equivalent to a "survey" in the standard occupancy-detection framework; MacKenzie et

158 al. 2002). For each collection event we defined

159

$$
h_{t}=\left\{\begin{array}{ll}
1 & \text { if collector } j \text { collected the focal species at last once in county } i \text { on month } m \\
0 & \text { otherwise }
\end{array} .\right.
$$

160 For each collector we examined their collection history across all their records and tallied the

161 number of species and genera they collected, the months they collected in, and the number of

162 each of our focal species they collected.

163

164

165

166

172 where $x_{1}$ represented human population density; $x_{2}$ average annual minimum monthly

173 temperature; and $x_{3}$ county area. Population density was obtained from Recht (2019) and

174 minimum temperature from 2-arcmin PRISM climate data for 1895 through 2019 (Daly et al.

175 2002). All covariates were z-standardized (MacKenzie et al. 2018). 
176 The occupancy status $Z_{i}$ of site $i$ is a latent state that we cannot directly measure. Instead,

177 through the observation process we measure $y_{i, t}$, whether a detection of the species occurs in

178 collection event $t$. This observation process was modeled as:

$$
y_{i, t} \mid Z_{i} \sim \operatorname{Bernoulli}\left(p_{i, t} \cdot Z_{i}\right)(\text { Eq. 3) }
$$

180

181

182

183

184

185

186

187

188

189

190

191

192

193

194

where $p_{i, t}$ is the probability of detecting the species given that it is present. In the simple models, each county is assumed to have the same baseline probability of collection $p$, which has a flat beta prior. For the full models, the collection probability $p$ depends on several collection-specific covariates,

$$
\operatorname{logit}\left(p_{i, t}\right)=\epsilon_{c o l}+\epsilon_{m o s}+\epsilon_{y r}+\beta_{1} x_{4}+\beta_{2} x_{5}(\text { Eq. } 4)
$$

where $\epsilon_{c o l}, \epsilon_{m o s}$, and $\epsilon_{y r}$ are random effects associated with collector, month and year, respectively. Since collectors are less likely to collect the same species in the same place twice (ter Steege et al. 2011), we defined a binary variable, $x_{4}$, to designate whether or not a collector had previously collected the focal species in that county before. Variable $x_{5}$ indicated whether the collector was a citizen scientist $\left(x_{5}=1\right)$ or an herbarium collector $\left(x_{5}=0\right)$. The full likelihoods and priors for both the simple and full model are available in Appendix B.

To evaluate the effectiveness of data filtration (Fig. 1a), we ran both the simple and full models first using the full set of detection histories (all data) and then using a subset of the data excluding collection events from collectors who only collected a single genus and never collected the focal species (filtered data).

For each of the four combinations (full model with all of the data, full model with filtered data, simple model with all of the data and simple model with filtered data), we ran two chains for 
197349000 iterations using the Nimble Bayesian modeling system version 0.9.0 (de Valpine et al.

198 2017) for R version 3.6.1 (R Core Team 2019), discarding the first 10000 iterations as burn-in.

199 We assessed convergence using the Gelman-Rubin diagnostic (R-hat) and visually inspected the

200 chains using the R package MCMCvis (Youngflesh 2018).

202 To assess the predictive accuracy of our models we used k-fold cross-validation with 5 folds for each of our species-model-data combinations. We assembled each fold by randomly dividing the

204 list of counties into one of five groups. Since estimating random effects (Eq. 4) requires that there is at least one observation for each collector, year and month, if any of the resulting training data sets (assembled from the remaining folds) was deficient in any of these categories, one of the occurrence records of that level in the held-out fold was randomly selected and moved from the held-out data into the training data set. On each of the 5 runs, one fold was held out, the remaining training data was used to run the models, and the resulting model was used to predict the held-out data points. We evaluated the ability of each of the models to predict the held-out

211 data using a scaled log-likelihood metric

$$
\begin{gathered}
L_{l o g}=-\frac{1}{k} \sum_{k} \sum_{s=1}^{S} \sum_{i=1}^{M} \sum_{j=1}^{J_{i}} \log \left[y_{k} \mid y_{-k}, \theta^{(s)}\right] \\
=\frac{1}{k} \sum_{k} \sum_{s=1}^{S} \sum_{i=1}^{M} \sum_{j=1}^{J_{i}} \frac{y_{i j} \log \left[\psi_{i}^{(s)} p_{i j}^{(s)}\right]}{N_{1}}+\frac{\left(1-y_{i j}\right) \log \left[1-\psi_{i}^{(s)} p_{i j}^{(s)}\right]}{J_{i}-N_{1}}(\text { Eq. 5) }
\end{gathered}
$$

214 where $k$ is the number of folds, $y_{i j}$ is the held-out data for the $i=1, \cdots, M$ counties, and $t=$

$2151, \cdots, J_{i}$ surveys, where $J_{i}$ represents the number of surveys of county $i$ in the held-out data,

216 evaluated over $S$ total MCMC iterations. To allow comparison between the filtered and unfiltered 
217 models, which have different numbers of non-detections of the focal species, we scaled the 218 respective likelihoods by the number of detections $N_{1}$ and the number of non-detections $\left(J_{i}-\right.$ $\left.219 N_{1}\right)$.

\section{RESULTS}

There were 762 people who collected at least a single Anacardiaceae species in Florida between the years 1895 and 2019, of whom 287 were citizen scientists and 475 herbarium collectors

224 (Figure 3d). The number of records collected by a single collector was highly skewed, with some 225 people collecting over 5000 records, while the most frequent number of records collected was one. There was temporal variation in the number of records collected by month as well as by

227 decade (for example, as can be seen in Figure 3b and c for M. toxiferum). Most collection events represented the first time a collector collected that species in the county (Figure 3e). The spatial

229 distribution of collections across Florida as a whole (Fig. 3h) and by collector, as can be seen, for 230 example, with the two collectors who collected the greatest number of Tracheophytes in Florida

231 (Figures $3 \mathrm{f}$ and $3 \mathrm{~g}$ ).

233 For each species, cross-validation demonstrated that the full model with collection covariates

234 using filtered non-detection data had the highest predictive accuracy (lowest log-likelihood

235 score) against withheld data, followed by the full model using all non-detection data, and then

236 lastly the two simple models. For six of the seven species, the simple model using all of the data

237 performed the worst (had the highest log-likelihood score, Figure 4). Each of the full models 238 performed better than the simple models, while the magnitude of the effect of data filtration 
239 varied across the species, improving model performance for some species, while having little

240 effect for others, indicating that including detection covariates improved predictive accuracy

241 more than filtering the data.

243 For each species, both of the full models that included collector covariates indicated that if a

244 collector had previously recorded the focal species, they were more likely to collect it again

245 (odds ratios ranging from 5.21 to 85.29 more likely, odds ratios calculated as $e^{\mu}$ where $\mu$ is the

246 mean of the posterior distribution of the parameter). Citizen scientists had a higher probability of

247 collecting $M$. toxiferum, R. copallina, S. terebinthifolia, and T. radicans (odds ratios from 2.50 to

248 10.38). Collectors who were preserving specimens on herbarium sheets were 3.23 and 94.28

249 times more likely to collect $M$. indica and $T$. pubescens, respectively, while $T$. vernix was only

250 collected by those making herbarium specimens.

251 Occupancy covariates

252 For each of the species, the four models mostly agreed with each other in terms of which

253 covariates were important for predicting occupancy. The posterior density for the coefficient

254 associated with population density overlapped zero for all of the models except the simple model

255 using filtered data for $M$. indica, which suggested a positive association between human

256 population density and occupancy. Minimum temperature was positively associated with

257 occupancy for $M$. indica, S. terebinthifolia, and negatively associated with occupancy for $T$.

258 pubescens and T. vernix for all four models for each species. County area was only relevant for

259 one species, with all four models suggesting a positive relationship between county area and

260 occupancy for T. vernix. (Appendix D Figure 1). 


\section{DISCUSSION}

263 The objectives of our study were to a) develop a framework for applying methods traditionally

264 applied to structured data (occupancy-detection modeling); and b) evaluate whether bias-

265 correction methods used in this framework and/or borrowed from species distribution modeling

266 improved predictive accuracy. We found that models with the highest predictive accuracy

267 incorporated both the background data filtration step inherited from the species distribution

268 modeling framework (Fig. 1a) as well as the incorporation of collector covariates associated with

269 the probability of collection from the occupancy-detection modeling framework (Figure 1b).

270 Including collector covariates improved model performance more than data filtration (Figure 4).

271 We found that for all species, individuals who had previously collected the focal species had a

272 much higher probability of collecting the species again (Figure 5a). Previous collection of the

273 focal species is one way of measuring the experience of individual collectors, and several studies

274 have indicated that observer experience is positively associated with increased detection

275 probability (i.e., Berberich et al. 2016, Johnston et al. 2018), although previous work also

276 suggests that individuals are more likely to collect species they have not previously collected (ter

277 Steege et al. 2011). In our study, most collectors never collected the focal species (ranging from

27858 to $96 \%$ of the 762 collectors).

279 We found species-specific differences in which type of collector (citizen scientists versus

280 botanical collectors) was more likely to collect the focal species. Four of the species were more

281 likely to be detected by citizen scientists (M. toxiferum, R. copallina, S. terebinthifolia, and T.

282 radicans), while the remaining species were either never collected by citizen scientists $(T$. 
283

284

285

286

287

288

289

290

291

292

293

294

295

296

297

298

299

300

301

302

303

304

vernix) or more likely to be preserved as herbarium specimens (M. indica and T. pubescens). In contrast to the process by which a citizen scientist typically records a plant (which usually involves taking a photograph and possibly annotating it), preparing an herbarium specimen is a more involved process and involves physically obtaining the specimen, mounting it to a specimen sheet, and (for our uses), deposition of the label data into an electronic data portal. There are therefore possibly species-specific traits which could influence the probability of collection according to whether the collection was performed by a citizen scientist or botanical collector. Indeed, all of the members of the Anacardiaceae produce the secondary compound urushiol, a skin irritant (Judd et al. 2008), which could dissuade collectors who need to physically handle the specimens. Species also differ with regard to how easy they are to identify or even notice by non-experts (i.e., Tulloch et al. 2013, Bird et al. 2014, Meyer et al. 2016, Johnston et al. 2018).

There are well-documented temporal biases in digital occurrence data (Sólymos 2007, Meyer et al. 2016), that are also present in our data set (Figure 3c). However, while we found that collection probability did vary across the months for many of the species (Figure 3b, Appendix E), we did not see evidence of a year effect on collection, even for introduced species (Appendix E), which suggests that some of our other covariates are correlated with the year. For example, citizen science campaigns focused on biodiversity are generally a more recent phenomenon, so the rate of collection by citizen scientists is positively associated with year. Likewise, we also found little to no evidence that including a separate random effect for each collector was necessary (Figure 5, Appendix E), which also suggests that our existing covariates are adequately capturing the existing variation among collectors.

\section{Occupancy-collection modeling}


Our work shows a clear advantage to modeling unstructured data with "structured" techniques which can explicitly account for bias. However, adapting occupancy-detection models for use with unstructured data requires careful consideration of model assumptions and requirements. These include populations closure, no un-modeled heterogeneity, how to handle false identifications, and choosing appropriate sampling units ( e.g. MacKenzie et al. 2018, Altwegg and Nichols 2019). Depending on the time-scale of the study and the life-history of the organism, there is a tradeoff between strictly satisfying the closure assumption versus limiting the interpretation of the resulting occupancy estimate as a measure of habitat "usage" probability (MacKenzie et al. 2018). Carefully considering sources of variation in both detection and occupancy probabilities, as well as including random effects can help ensure that all sources of heterogeneity in the data is accounted for. The issue of false identifications has been welldocumented in the citizen science literature (Ruiz-Gutierrez et al. 2016, Cruickshank et al. 2019), which can be addressed in the modeling process (Miller et al. 2011). Finally, a key decision of adapting unstructured data for use in an occupancy-detection framework requires careful consideration of how to define spatial units and surveys. We chose counties as our spatial units. However, it is possible that the same analysis could be replicated at smaller spatial scales (i.e., assigning records into grid cells). When determining the appropriate spatial scale to use and how to delineate what constitutes a survey, consideration should be made to ensure that the choice of these definitions results in each site having at least two independent visits.

We focused on collection covariates that were readily available and that we hypothesized could be relevant for all of the species we considered. In a smaller-scale study with fewer collectors, it would be possible to gather additional information about collectors that could provide a more complete picture. For example, the home institution of a given collector could provide additional 
329 information on where that person is most likely to collect. Leveraging information from

330 additional sources such as the Index Herbariorum (http://sweetgum.nybg.org/science/ih/) and

331 Bionomia (https://bionomia.net) that collate additional biographical information about collectors

332 could allow for the incorporation of additional information to further distinguish among

333 collectors. Harmonizing collector names across records and constructing collection histories is a

334 laborious process. Mobilizing this store of information on collection would be aided by

335 standardization of allowable entries in database fields, as well as advances in artificial

336 intelligence and textual analysis.

337 Conclusions

338 Our work is a test of how to account for widely-appreciated biases in unstructured data (Meyer et

modeling the distribution of species, and accounting for biases associated with the manner in

341 which the data was collected. Species distribution models, which make use of the ever-increasing

342 stream of digital biodiversity occurrence data, typically account for these biases through various

343 data filtration steps but fall short of modeling the processes that lead to the resulting biases in the

344 data (Figure 1a). On the other hand, occupancy-detection models explicitly model the role of

345 imperfect detection in the data that we are able to collect, but they do so in a way that assumes

346 the data come from structured repeat-visit surveys (Figure 1b). We found that utilizing both the

347 bias-correcting filtration techniques from species-distribution modeling while explicitly

348 modeling the manner in which the data was collected resulted in the best predictive accuracy. We

349 therefore need methods like those explored in this study (Figure 1c), to allow for the use of

350 digital occurrence data while explicitly accounting for the imperfect-detection processes that led

351 to their existence. 
DATA AVAILABILITY STATEMENT

354 Upon acceptance, data will be deposited in an online repository.

\section{ACKNOWLEDGEMENTS}

356 The authors thank Stephen Murphy, James Lucas, David Henderson, Matt Austin, and the

357 Stephen Beissinger lab at UC Berkeley for helpful discussion and feedback.

This project was made possible in part by the Institute of Museum and Library Services (FAIN

MG-30-15-0094-15) and the Alan Graham Fund in Global Change.

\section{REFERENCES}

362

363

364

365

366

367

368

369

370

371

Altwegg, R. and Nichols, J. D. 2019. Occupancy models for citizen-science data. - Methods Ecol. Evol. 10: 8-21.

Berberich, G. M. et al. 2016. Detection probabilities for sessile organisms. - Ecosphere 7: e01546.

Bird, T. J. et al. 2014. Statistical solutions for error and bias in global citizen science datasets. Biol. Conserv. 173: 144-154.

Bled, F. et al. 2013. Dynamic occupancy models for analyzing species' range dynamics across large geographic scales. - Ecol. Evol. 3: 4896-4909.

Boria, R. A. et al. 2014. Spatial filtering to reduce sampling bias can improve the performance of ecological niche models. - Ecol. Modell. 275: 73-77. 
372 Briscoe, N. J. et al. 2019. Forecasting species range dynamics with process-explicit models:

Brooks, T. M. et al. 2004. Protected areas and species. - Conserv. Biol. 18: 616-618.

375

376

377

378

379

380

381

382

Collins, S. D. et al. 2017. Quantifying the degree of bias from using county-scale data in species distribution modeling: Can increasing sample size or using county-averaged environmental data reduce distributional overprediction? - Ecol. Evol. 7: 6012-6022.

Cruickshank, S. S. et al. 2019. Quantifying data quality in a citizen science monitoring program: False negatives, false positives and occupancy trends. - Conserv. Sci. Pract. 1: 1-14.

Daly, C. et al. 2002. A knowledge-based approach to the statistical mapping of climate. - Clim. Res. 22: 99-113.

de Valpine, P. et al. 2017. Programming models: writing statistical algorithms for general model structures with NIMBLE. - J. Comput. Graph. Stat. 26: 403-413.

Fourcade, Y. et al. 2014. Mapping species distributions with MAXENT using a geographically biased sample of presence data: A performance assessment of methods for correcting sampling bias. - PLoS One 9: 1-13.

Graham, L. J. et al. 2017. Future geographic patterns of novel and disappearing assemblages across three dimensions of diversity: A case study with Ecuadorian hummingbirds. - Divers. Distrib. 23: 944-954.

Hijmans, R. J. et al. 2000. Assessing the geographic representativeness of genebank collections: The case of Bolivian wild potatoes. - Conserv. Biol. 14: 1755-1765.

Huynh, D. and Mazzocchi, S. 2019. OpenRefine. 
Johnston, A. et al. 2018. Estimates of observer expertise improve species distributions from citizen science data. - Methods Ecol. Evol. 9: 88-97.

Judd, W. S. et al. 2008. Plant systematics: a phylogenetic approach. - W. H. Freeman.

Louvrier, J. et al. 2018. Mapping and explaining wolf recolonization in France using dynamic occupancy models and opportunistic data. - Ecography (Cop.). 41: 647-660.

MacKenzie, D. I. et al. 2002. Estimating site occupancy rates when detection probabilities are less than one. - Ecology 83: 2248-2255.

MacKenzie, D. I. et al. 2003. Estimating site occupancy, colonization, and local extinction when a species is detected imperfectly. - Ecology 84: 2200-2207.

MacKenzie, D. I. et al. 2018. Occupancy Estimation and Modeling: Inferring Patterns and Dynamics of Species Occurrence. - Academic Press.

Meineke, E. K. et al. 2018. The unrealized potential of herbaria for global change biology. Ecol. Monogr. 88: 505-525.

Meyer, C. et al. 2016. Multidimensional biases, gaps and uncertainties in global plant occurrence information. - Ecol. Lett. 19: 992-1006.

Miller, D. A. et al. 2011. Improving occupancy estimation when two types of observational error occur: non-detection and species misidentification. - Ecology 92: 1422-1428.

Miller, R. A. et al. 2016. Short-eared Owl (Asio flammeus) surveys in the North American Intermountain West: utilizing citizen scientists to conduct monitoring across a broad geographic scale. - Avian Conserv. Ecol. 11: art3.

Park, D. S. and Davis, C. C. 2017. Implications and alternatives of assigning climate data to 
geographical centroids. - J. Biogeogr. 44: 2188-2198.

415

416

417

418

419

420

421

422

423

424

425

426

427

428

429

430

431

432

433

434

Pender, J. E. et al. 2019. How sensitive are climatic niche inferences to distribution data sampling? A comparison of Biota of North America Program (BONAP) and Global Biodiversity Information Facility (GBIF) datasets. - Ecol. Inform. 54: 100991.

Peterson, A. T. et al. 2011. Ecological Niches and Geographic Distributions. - Princeton University Press.

Phillips, S. J. et al. 2009. Sample selection bias and presence-only distribution models: Implications for background and pseudo-absence data. - Ecol. Appl. 19: 181-197.

Ponder, A. W. F. et al. 2001. Evaluation of Museum Collection Data for Use in Biodiversity Assessment Linked references are available on JSTOR for this article : Evaluation of Museum Collection Data for Use in Biodiversity Assessment. 15: 648-657.

Powney, G. D. and Isaac, N. J. B. 2015. Beyond maps: A review of the applications of biological records. - Biol. J. Linn. Soc. 115: 532-542.

R Core Team 2019. R: A language and environment for statistical computing. - R Found. Stat. Comput. Vienna, Austria

Recht, H. 2019. censusapi: Retrieve Data from the Census APIs. R package version 0.7.1.

$$
\text { https://CRAN.R-project.org/package=censusapi }
$$

Requena-Mullor, J. M. et al. 2019. Integrating anthropogenic factors into regional-scale species distribution models - A novel application in the imperiled sagebrush biome. - Glob. Chang. Biol.

Roberts, R. L. et al. 2007. Using simple species lists to monitor trends in animal populations: 
New methods and a comparison with independent data. - Anim. Conserv. 10: 332-339.

436

437

438

439

440

441

442

443

444

445

446

447

448

449

450

451

452

453

454

455

Ruiz-Gutierrez, V. et al. 2016. Uncertainty in biological monitoring: a framework for data collection and analysis to account for multiple sources of sampling bias. - Methods Ecol. Evol. 7: 900-909.

Scherrer, D. et al. 2017. Assessing and predicting shifts in mountain forest composition across 25 years of climate change. - Divers. Distrib. 23: 517-528.

Sólymos, P. 2007. Geographic and taxonomic bias in land snail distribution data of Hungary. Community Ecol. 8: 239-246.

ter Steege, H. et al. 2011. A model of botanical collectors' behavior in the field: Never the same species twice. - Am. J. Bot. 98: 31-37.

Tulloch, A. I. T. et al. 2013. To boldly go where no volunteer has gone before: Predicting volunteer activity to prioritize surveys at the landscape scale. - Divers. Distrib. 19: 465-480.

Václavík, T. T. and Meentemeyer, R. K. 2012. Equilibrium or not? Modelling potential distribution of invasive species in different stages of invasion. - Divers. Distrib. 18: 73-83.

Van der Weyde, L. K. et al. 2018. Multi-species occupancy modelling of a carnivore guild in wildlife management areas in the Kalahari. - Biol. Conserv. 220: 21-28.

Van Strien, A. J. et al. 2013. Opportunistic citizen science data of animal species produce reliable estimates of distribution trends if analysed with occupancy models. - J. Appl. Ecol. 50: $1450-1458$.

Varela, S. et al. 2014. Environmental filters reduce the effects of sampling bias and improve predictions of ecological niche models. - Ecography (Cop.). 37: 1084-1091. 
456 Veran, S. et al. 2016. Modeling spatial expansion of invasive alien species: Relative

457 contributions of environmental and anthropogenic factors to the spreading of the harlequin

458 ladybird in France. - Ecography (Cop.). 39: 665-675.

459 Vollering, J. et al. 2019. Bunching up the background betters bias in species distribution models.

$460 \quad$ - Ecography (Cop.). 42: 1717-1727.

461 Wolf, A. et al. 2011. Robust detection of plant species distribution shifts under biased sampling

462 regimes. - Ecosphere 2: art115.

463 Youngflesh, C. 2018. MCMCvis: Tools to visualize, manipulate, and summarize MCMC output.

$464 \quad$ Journal of Open Source Software 3 (24) 640. doi: 10.21105/joss.00640 (URL:

465

https://doi.org/10.21105/joss.00640)

466 Zurell, D. et al. 2016. Benchmarking novel approaches for modelling species range dynamics. -

467 Glob. Chang. Biol. 22: 2651-2664. 
(a) Species

Distribution Models

477 (b) OccupancyDetection Models

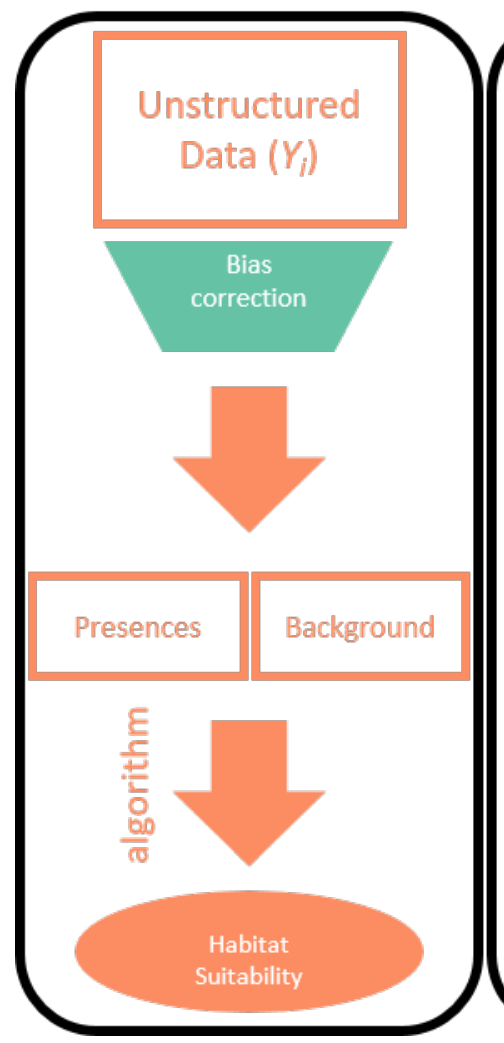

(c) OccupancyCollection Models

\section{P(Presence)}

P(Presence)

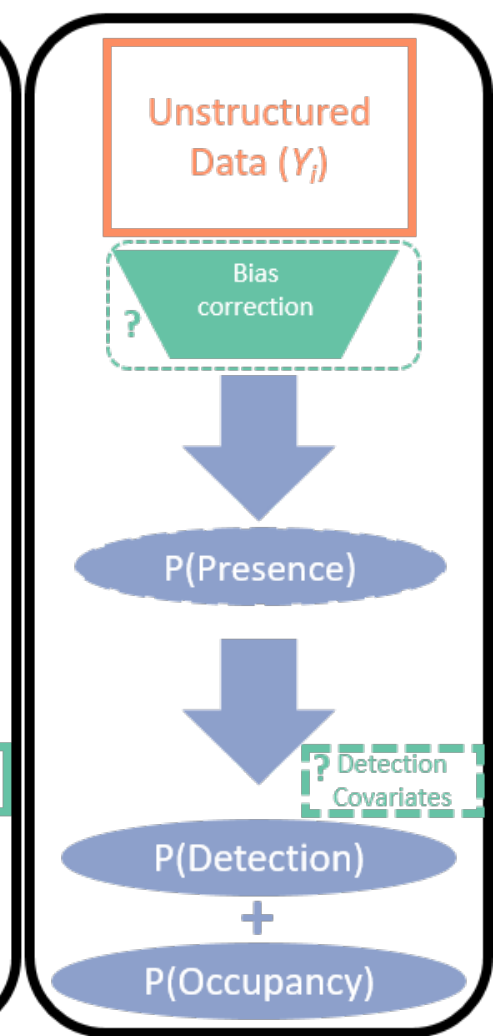

$\mathrm{P}$ (Detection)

P(Detection) $+$ P(Occupancy)

Figure 1. Schematic of modeling approaches for predicting species distributions: (a) species distribution models, which are frequently used to predict habitat suitability using unstructured occurrence data (i.e., museum or herbarium data), with little to no accounting for the processes underlying how the data was collected; (b) occupancy-detection models, which account for imperfect detection probabilities for systematic repeated-visit sampling; and (c) the modeling approach we introduce in this paper, which makes use of the unstructured occurrence data while accounting for the mechanism of collection. Colorblind-friendly colors from www.ColorBrewer.org by Cynthia A. Brewer, Geography, Pennsylvania State University. 
(a)

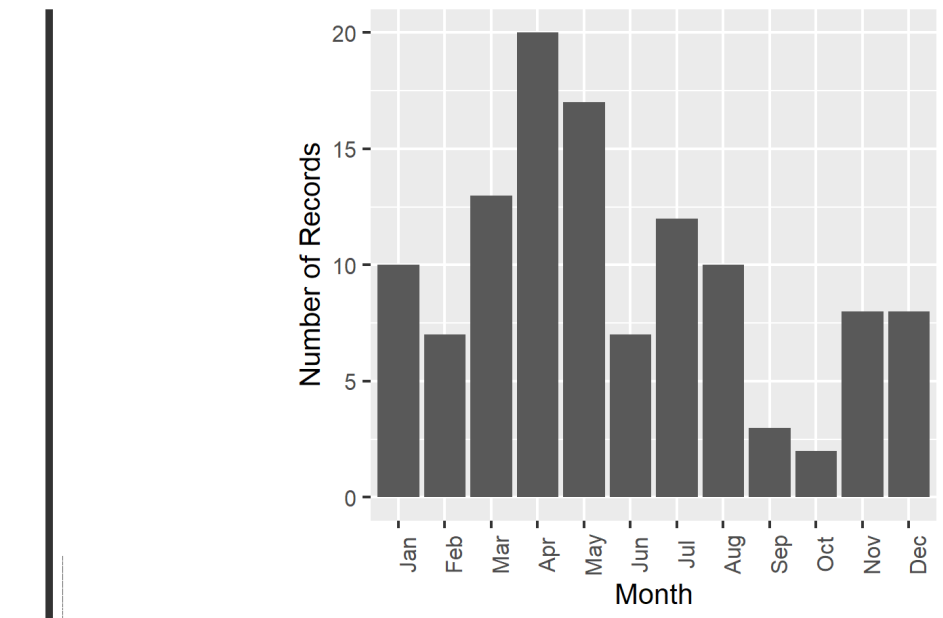

(d)

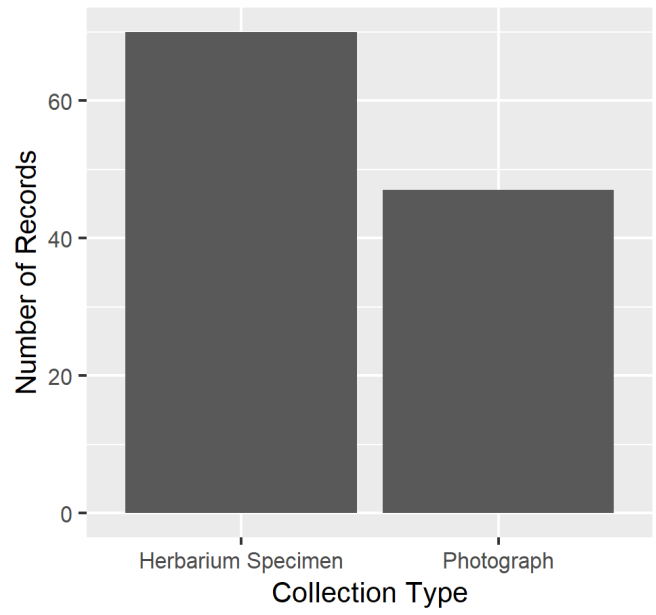

(f)

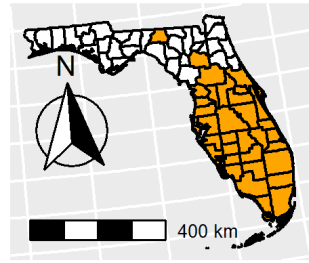

(c)

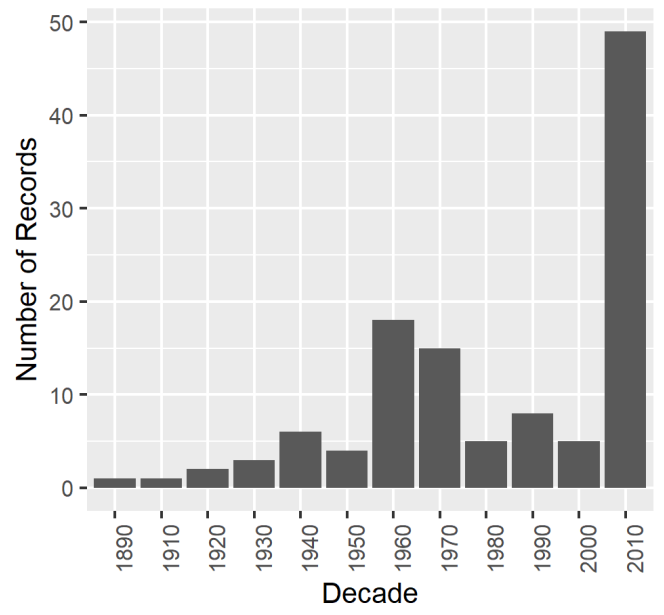

(e)

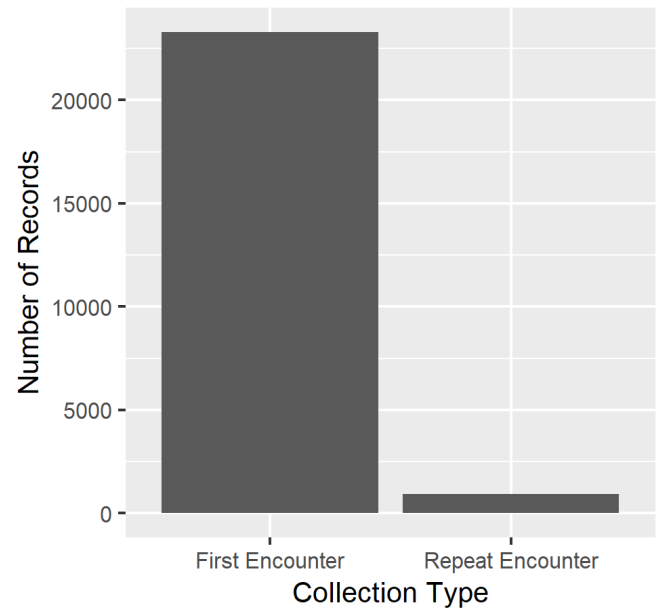

(h)

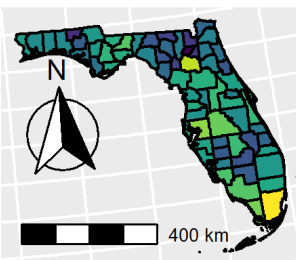

$\log$ (Number of Records)

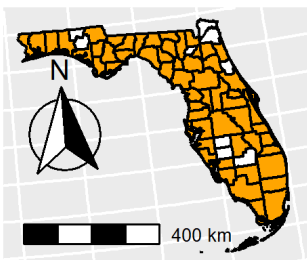


487 Figure 2. Biases in collection behavior. (a) Histogram of the number of records collected by each

488 collector. Most collectors collect only one specimen. (b) Number of records of Metopium

489 toxiferum collected in each month of the year. (c) Number of records of $M$. toxiferum collected in

490 each decade. (d) Number of records of M. toxiferum that are deposited in an herbarium or a

491 citizen science data portal. (e) Number of records of $M$. toxiferum that represent first encounters

492 or repeat encounters. (f) Counties visited by the second-most prolific collector of specimens of

493 Tracheophytes in Florida (O. K. Lakela). (g) Counties visited by the most prolific collector (J. R.

494 Abbott). (h) Spatial map of collection effort (number of records of all species in the

495 Tracheophytes) in each county in Florida on a $\log _{10}$ scale.

496

497 
(a)

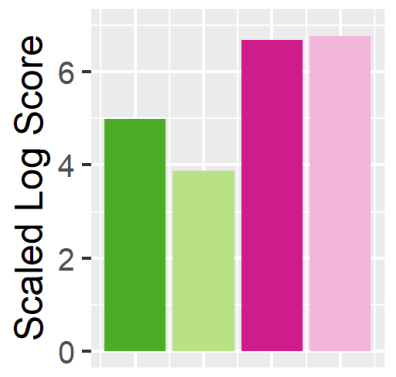

(e)

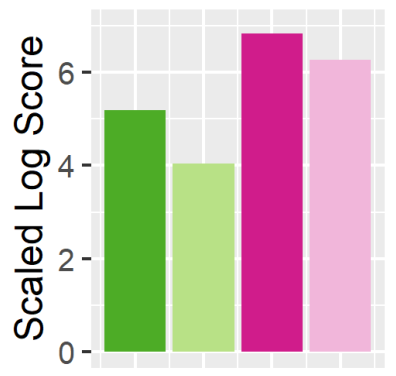

(b)

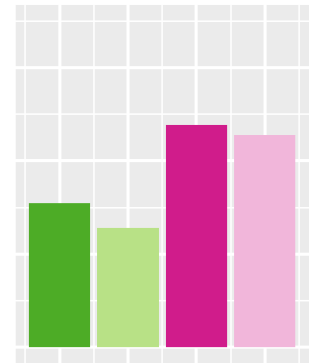

(f)

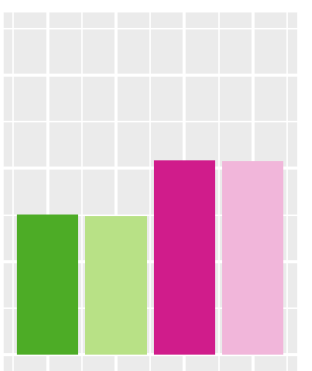

(c)

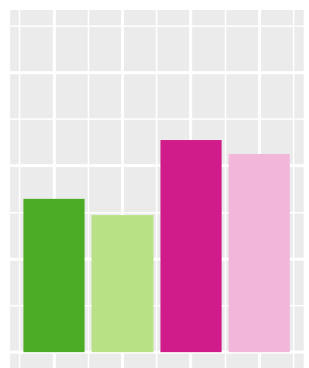

(g)

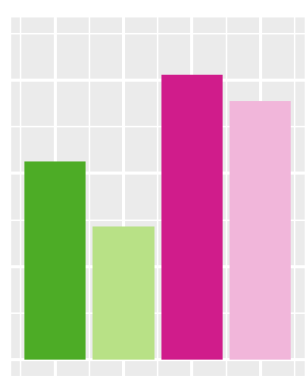

(d)

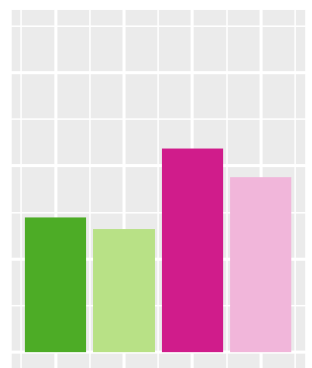

Method

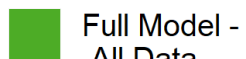

All Data

Full Model -

Filtered

Simple Model -

All Data

Simple Model -

Filtered

502 Figure 3. Cross-validation scores for the full models with all data (dark green), full models with

503 filtered data (light green), simple models with all data (dark pink), and simple models with

504 filtered data (light pink) for (a) M. indica, (b) M. toxiferum, (c) R. copallina, (d) $S$.

505 terebinthifolia, (e) T. pubescens, (f) T. radicans, and (g) T. vernix. Smaller values indicate better

506 predictive accuracy. Colorblind-friendly colors from www.ColorBrewer.org by Cynthia A.

507 Brewer, Geography, Pennsylvania State University. 
(a)

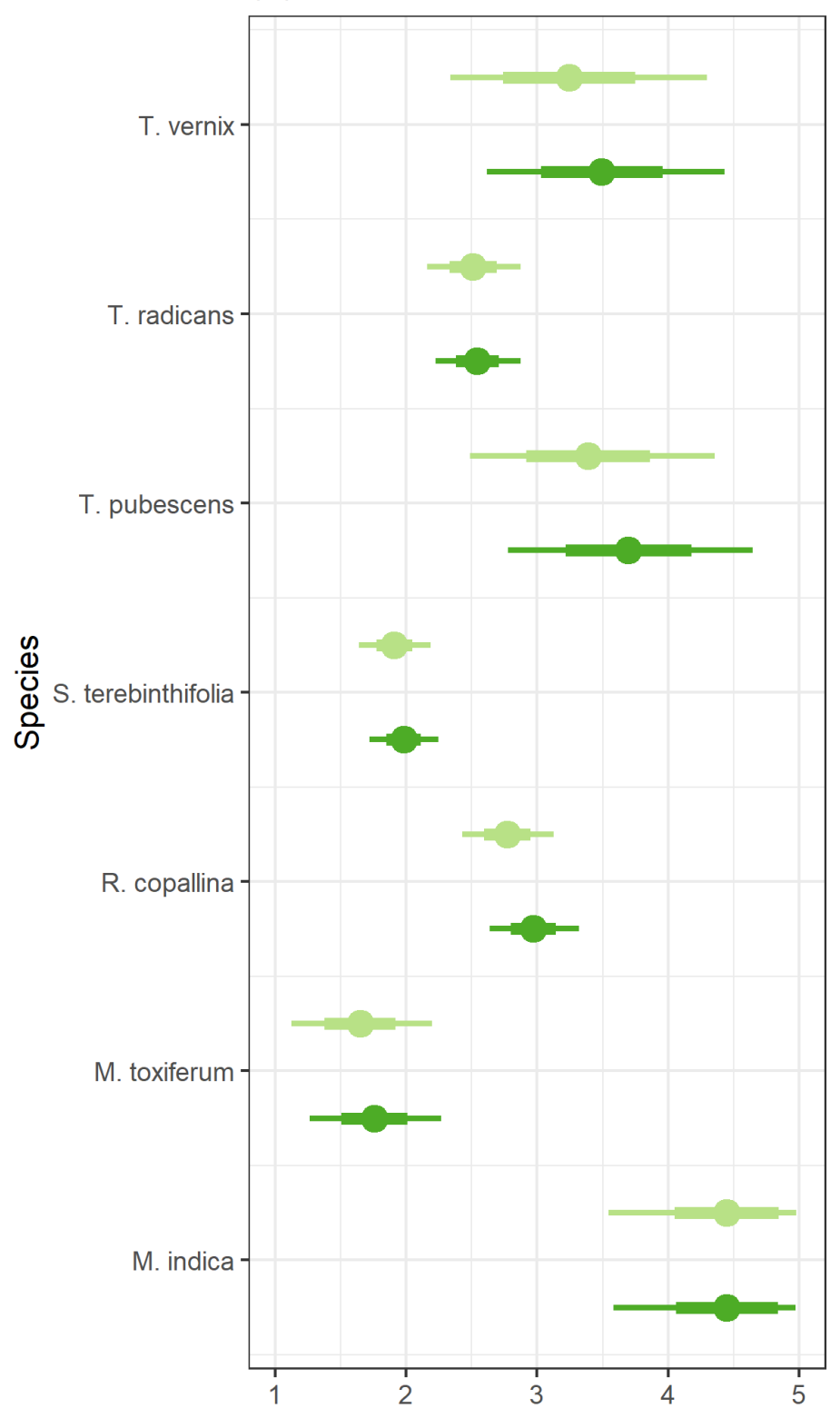

(b)

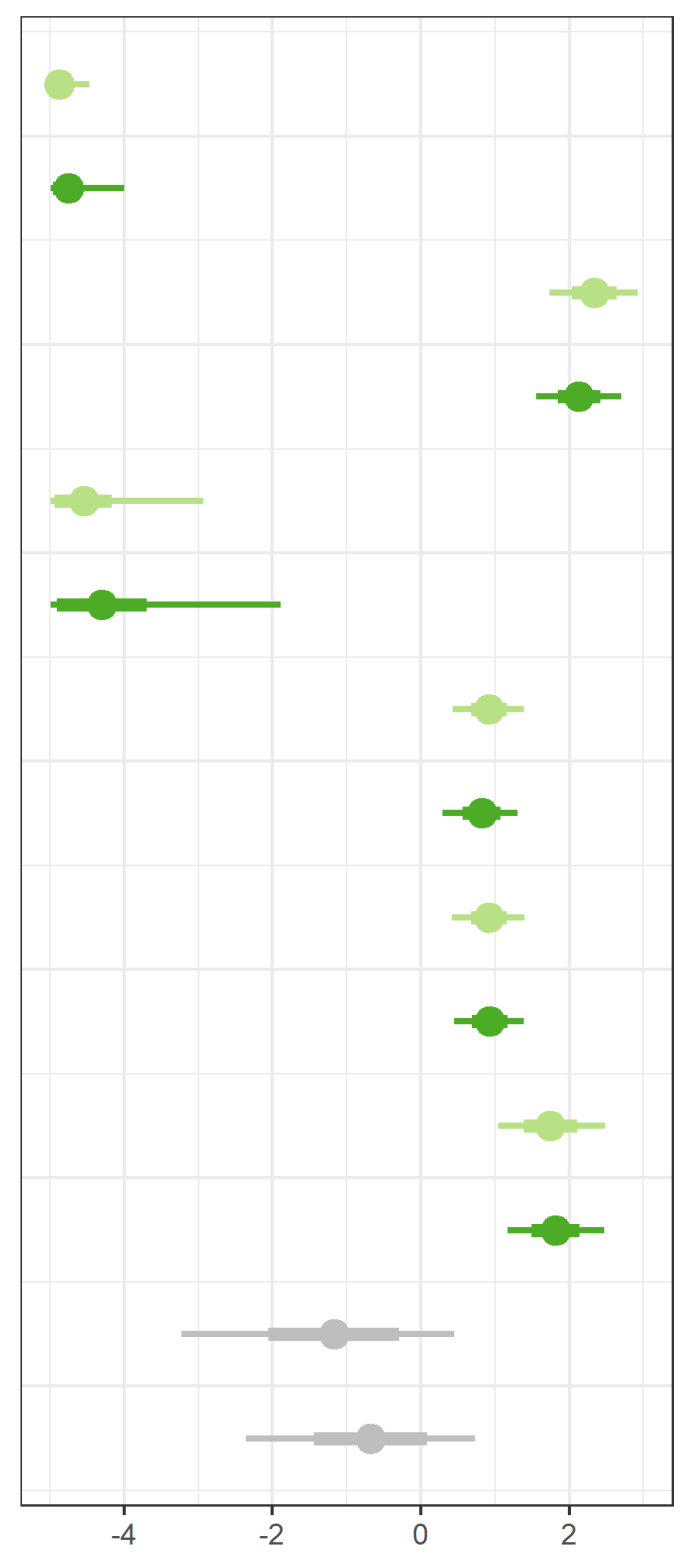

509 Figure $4.50 \%$ (thin line) and 95\% (thick line) posterior credible intervals for (a) detection

510 history and (b) type of collection (photograph or herbarium specimen). For each species, the

511 bottom line (dark green) is the credible interval for the parameter estimate from the full model

512 with all data and the top line (light green) is the credible interval for the parameter estimate from

513 the full model with filtered data. Grey lines indicate that the $95 \%$ credible interval overlaps with 
bioRxiv preprint doi: https://doi.org/10.1101/2021.01.06.425644; this version posted January 8, 2021. The copyright holder for this preprint

(which was not certified by peer review) is the author/funder, who has granted bioRxiv a license to display the preprint in perpetuity. It is made available under aCC-BY-NC-ND 4.0 International license.

514 zero. Colorblind-friendly colors from www.ColorBrewer.org by Cynthia A. Brewer, Geography,

515 Pennsylvania State University. 
bioRxiv preprint doi: https://doi.org/10.1101/2021.01.06.425644; this version posted January 8, 2021. The copyright holder for this preprint (which was not certified by peer review) is the author/funder, who has granted bioRxiv a license to display the preprint in perpetuity. It is made available under aCC-BY-NC-ND 4.0 International license.

(a)

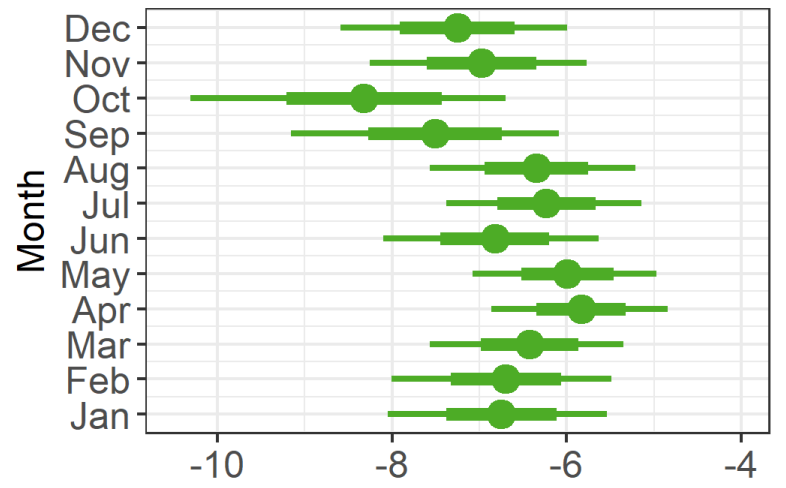

(c)

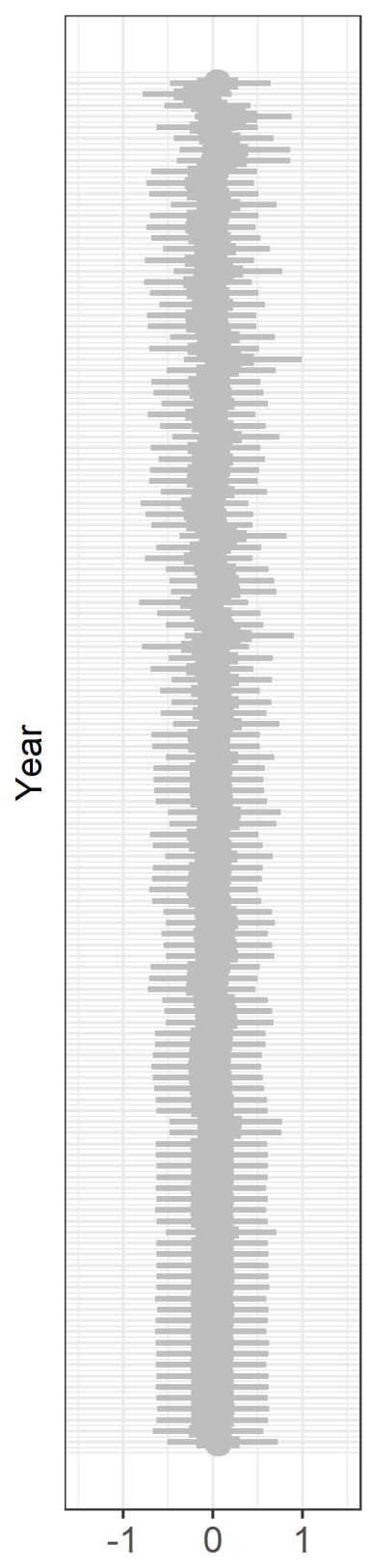

(d)

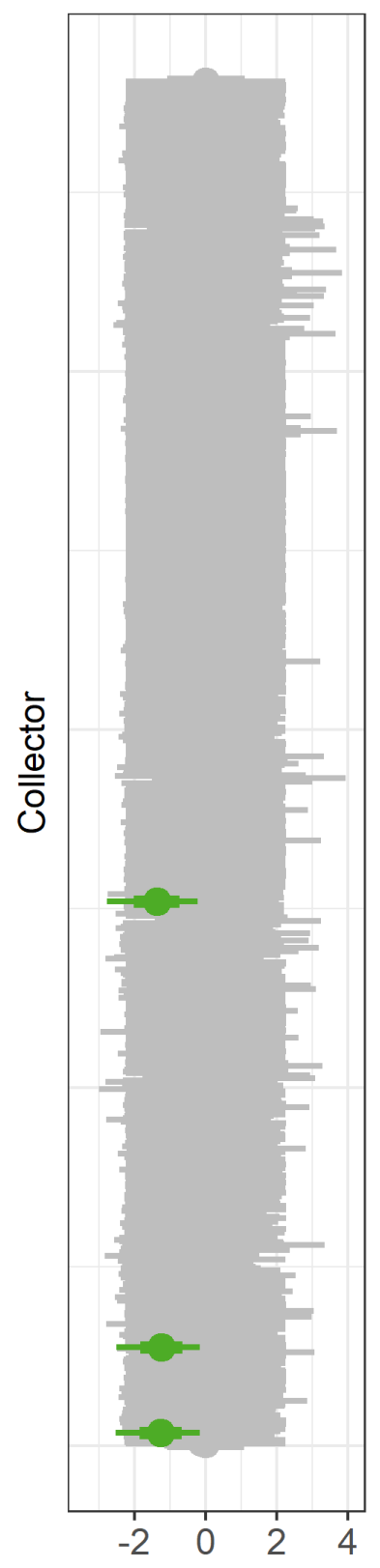

(b)

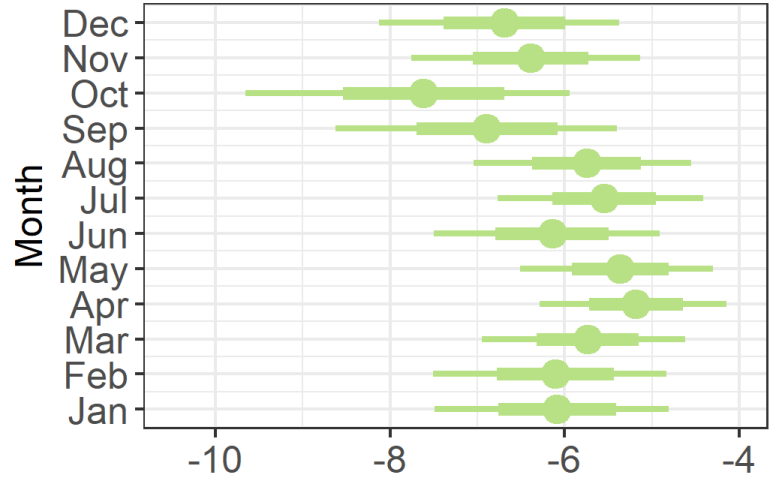

(e)

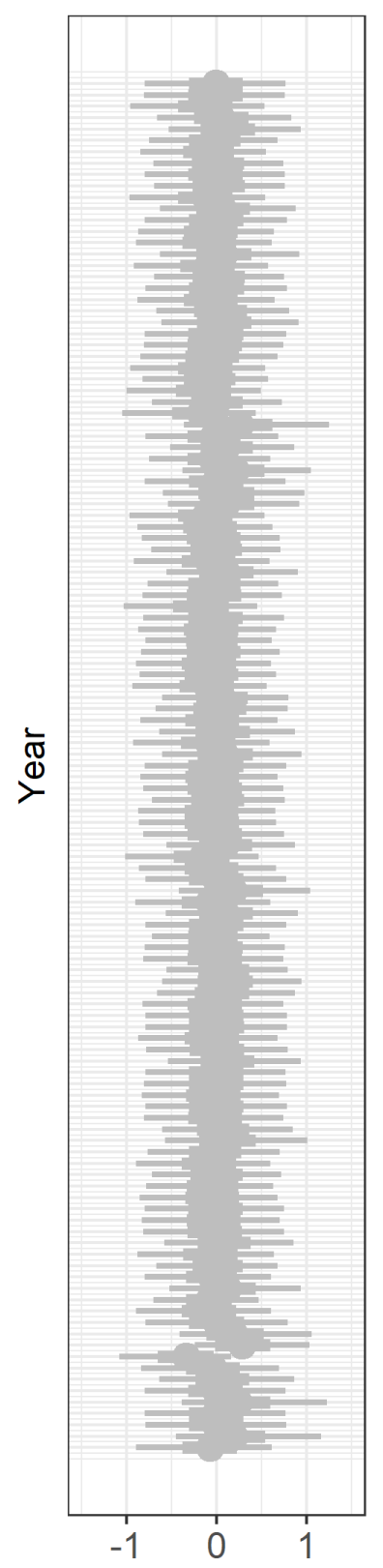

(f)

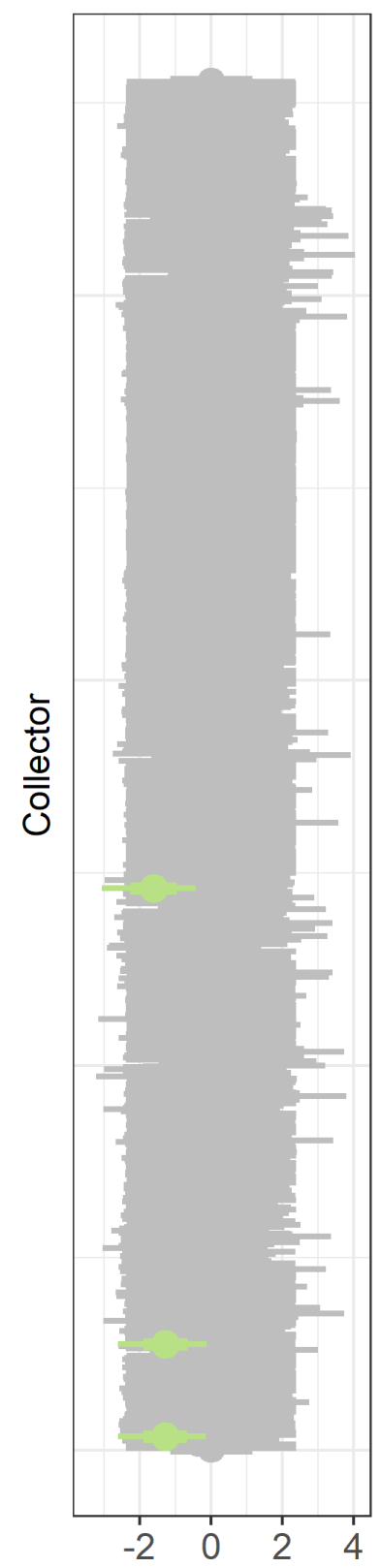


517 Figure 5. M. toxiferum 50\% (thick line) and 95\% (thin line) credible intervals for (a) month

518 effect for the full model with all data, (b) month effect for the full model with filtered data, (c)

519 collector effect for the full model with all data (d) year effect for the full model with all data (e)

520 collector effect for the full model with filtered data, and (f) year effect for the full model with

521 filtered data. Greyed-out credible intervals overlap 0. Colorblind-friendly colors from

522 www.ColorBrewer.org by Cynthia A. Brewer, Geography, Pennsylvania State University. 